\title{
Avaliações hematológicas e bioquímicas do uso de diclofenaco de sódio, meloxicam e firocoxibe em ratos
}

\author{
Evaluations hematological and biochemical by the use of sodium diclofenac, meloxicam and \\ firocoxib in rats
}

\author{
Cristiane Moraes BARBOSA ${ }^{1}$; Michiko SAKATE ${ }^{1}$; Annelise Carla CAMPLESI ${ }^{1}$; Maria do Carmo \\ Fernandez VAILATI ${ }^{1}$; Lívia Fagundes MORAES ${ }^{1}$; Regina Kiomi TAKAHIRA $^{1}$
}

${ }^{1}$ Faculdade de Medicina Veterinária e Zootecnia da Universidade Estadual Paulista, Botucatu-SP

\begin{abstract}
Resumo
O presente trabalho avaliou os parâmetros hematológicos e bioquímicos do uso de diclofenaco de sódio, meloxicam e firocoxibe em ratos Wistar. Os ratos foram distribuídos em grupos: G1 (controle), G2 (diclofenaco de sódio: $15 \mathrm{mg} / \mathrm{kg}$ ), G3 (meloxicam: 2,0 mg/ kg), G4 (meloxicam: 10,0 mg/ kg), G5 (firocoxibe: 5,0 mg/ kg) e G6 (firocoxibe: 25,0 mg/ kg). Os fármacos foram administrados por via intragástrica (gavage) a cada 24 horas, durante cinco dias e avaliados em três momentos: M1 (48 horas após o início do tratamento), M2 (96 horas após o início do tratamento) e M3 (72 horas após o término do tratamento). Em cada momento de cada grupo, foram avaliados de cinco a sete animais e realizados os exames laboratoriais. Não foram observadas alterações significativas nos parâmetros bioquímicos e hematológicos com o uso de meloxicam e firocoxibe. O diclofenaco de sódio produziu alterações no eritrograma (redução de hemácias, hematócrito e na taxa de hemoglobina) durante o tratamento e não alterou a contagem das plaquetas e leucometria, com exceção dos basófilos. Não produziu alterações nas atividades de AST, FA, GGT, ureia, creatinina, sódio e potássio. Entretanto, causou diminuições das proteínas plasmática e total sérica, albumina e globulina. Conclui-se que o diclofenaco de sódio não produz grandes alterações no hemograma e exames bioquímicos, enquanto que, o meloxicam e o firocoxibe não produzem alterações e efeitos deletérios dose-dependentes nestes exames laboratoriais.
\end{abstract}

Palavras-chave: Diclofenaco de sódio. Meloxicam. Firocoxibe. Exames laboratoriais. Ratos.

\begin{abstract}
This work has evaluated the hematological and biochemical profile by the use of sodium diclofenac, meloxicam and firocoxib in Wistar rats. The rats were distributed in groups: G1 (control), G2 (diclofenac sodium: $15 \mathrm{mg} / \mathrm{kg}$ ), G3 (meloxicam: $2.0 \mathrm{mg} / \mathrm{kg}$ ), G4 (meloxicam: $10.0 \mathrm{mg} / \mathrm{kg}$ ), G5 (firocoxib: $5.0 \mathrm{mg} / \mathrm{kg}$ ) e G6 (firocoxib: $25.0 \mathrm{mg} / \mathrm{kg}$ ). The drugs were administered intragastrically (gavage) once a day, during five days and evaluated in three moments: M1 (48 hours after the beginning of the treatment), M2 (96 hours after the beginning of the treatment) and M3 (72 hours after the ending of the treatment). In each moment of each group, five to seven animals were evaluated and laboratory exams were performed. There were no significant changes observed in the biochemical and hematological parameters by the use of meloxicam and firocoxib. One of the effects of the sodium diclofenac was eritrogram variation as hematocrit, erythrocytes, hemoglobin decrease during the treatment. In addition, the platelets and total white blood cells counts did not change except for basophil. There was no changes in AST, ALP, GGT, urea, creatinine, sodium, potassium values. However, the values of protein, globulin and albumin decreased. It was concluded that diclofenac sodium does not provide large variations in the hemogram and biochemical profile than the meloxicam and firocoxib do not provide delletery effects in laboratories tests.
\end{abstract}

Keywords: Sodium diclofenac. Meloxicam. Firocoxib. Laboratories parameters. Rats.

\section{Introdução}

Os agentes anti-inflamatórios não esteroides são extensamente utilizados pelos seres humanos e animais ${ }^{1}$. A ação dos AINES deve-se à inibição das enzimas ciclooxigenases (COXs), que convertem o ácido araquidônico, liberado das membranas fosfolipídicas,
Correspondência para:

Prof. Ass. Dra. Michiko Sakate

Universidade Estadual Paulista - UNESP/ Botucatu, Faculdade de

Medicina Veterinária e Zootecnia (FMVZ) - Departamento de Clínica

Veterinária, Distrito Rubião Júnior, sem número. Botucatu-SP.

CEP: $18618-000$

e-mail: michikos@fmvz.unesp.br

Recebido para publicação: 04/10/2008

Aprovado para publicação: 27/08/2009 
em prostaglandinas $(\mathrm{PGs})^{2,3}$. A enzima COX está presente em duas isoformas, COX-1 e COX- $2{ }^{4}$. A COX1 , presente na maioria dos tecidos, está relacionada à função renal, à agregação plaquetária e à proteção da mucosa gástrica ${ }^{5}$. Por outro lado, a enzima COX-2 tem sua expressão aumentada principalmente em processos inflamatórios, em resposta a mediadores da inflamação ${ }^{3}$. A toxicidade dos anti-inflamatórios não esteroidais (AINES) depende, entre diversos fatores, da sua ação mais ou menos seletiva sobre as COX-1 e COX $-2^{2}$.

Atualmente, há mais de cinquenta diferentes AINES no mercado e há ainda um fluxo contínuo de novas preparações ${ }^{6}$. O diclofenaco de sódio promove inibição inespecífica das COXs. Esta droga é bastante utilizada na medicina humana ${ }^{1}$, mas sua aplicação tem sido limitada devido a seus efeitos colaterais, que incluem lesões gastrointestinais, renais e hepáticas ${ }^{7}$.

O meloxicam é um AINE preferencialmente seletivo para COX-2 $2^{8}$ e tem se mostrado mais seguro em relação aos efeitos adversos, quando comparado aos dos anti-inflamatórios sem ação seletiva sobre a COX- $2^{9}$. Não induziu importantes mudanças nos parâmetros hematológicos e bioquímicos quando administrado em ratos ${ }^{10} \mathrm{e}$ em cães clinicamente saudáveis ${ }^{9}$. Promove efeitos deletérios, dose-dependentes, nas células sanguíneas em cães, quando administrado em doses cinco a dez vezes maiores que a dose terapêutica, durante 16 dias de tratamento ${ }^{11}$.

O firocoxibe é um inibidor altamente seletivo da COX-2 e é membro da classe coxibe desenvolvida especialmente para uso veterinário ${ }^{12}$. É aprovado pelo FDA (Food and Drug Administration) para controle da dor e inflamação causadas pela osteoartrite em cavalos e cães ${ }^{13}$. Apresentou boa tolerância e não induziu alterações hematológicas e bioquímicas em seis cães sadios que receberam firocoxibe durante 28 dias $^{14}$.

A existência de poucos trabalhos na comparação dos AINES de acordo com a sua capacidade de inibição COX- 1 e 2, em relação às alterações laboratoriais, e a ausência de dados sobre o novo anti-inflamatório firocoxibe em roedores, sustentam a proposta do presente estudo em avaliar as alterações hematológicas e bioquímicas do uso de diclofenaco de sódio, meloxicam e firocoxibe em ratos.

\section{Material e Método}

Foram utilizados 94 ratos Wistar, machos, de dois meses de idade e peso corporal entre 230 e 280 gramas. Os animais foram mantidos em gaiolas coletivas no biotério de experimentação, em ambiente com controle de luz (ciclos de 12 horas) e temperatura constante $\left(25^{\circ} \mathrm{C}\right)$, umidade do ar de $55-65 \%$, tempo de exaustão de dez trocas de ar da sala/hora, recebendo dieta padrão para animais de laboratório (Purina ${ }^{\circledast}$ ) e água ad libitum. Este estudo foi aprovado pela Câmara de Ética em Experimentação ( $n^{0}$ 80/2007). Os animais foram distribuídos em seis grupos: $(G)$ experimentais: G1-controle: $0,3 \mathrm{~mL} / 100 \mathrm{~g}$ de peso vivo de solução fisiológica; G2- diclofenaco de sódio (Voltaren ${ }^{\oplus}$, Novartis): 15 mg/kg; G3- meloxicam (Meloxicam $^{\oplus}$, EMS): $2,0 \mathrm{mg} / \mathrm{kg}$; G4- meloxicam: $10 \mathrm{mg} /$ kg; G5- firocoxibe (Previcox ${ }^{\oplus}$, Merial): $5,0 \mathrm{mg} / \mathrm{kg}$; G6- firocoxibe: $25,0 \mathrm{mg} / \mathrm{kg}$. Os animais foram avaliados em três momentos: (M): M1 (48 horas após o início do tratamento), M2 (96 horas após o início do tratamento) e M3 (72 horas após o término do tratamento). Em cada momento de cada grupo, foram avaliados cinco animais, com exceção do G1 em M1 e M2 $(n=6)$ e G2 em M3 ( $n=7)$. As dosagens do $\mathrm{G} 2^{15}$ e G3 ${ }^{16,17}$ foram estabelecidas com base em estudos experimentais. Não há estudos experimentais relatando o uso do firocoxibe em roedores, portanto a dosagem do G5 foi a mesma recomendada para cães ${ }^{14}$. As dosagens dos grupos G4 e G6 correspondem a cinco vezes às dosagens utilizadas respectivamente nos grupos G3 e G5. Os AINES foram remanipulados e o excipiente utilizado foi inerte. A dosagem de cada cápsula para cada grupo foi baseada no peso de 300 gramas. $\mathrm{O}$ conteúdo da cápsula foi diluído em $0,9 \mathrm{~mL}$ 
de solução fisiológica $0,9 \%$. Os ratos foram pesados e posteriormente calculou-se o volume de administração. A administração do AINE foi por via intragástrica pelo método de gavage $^{18}$, a cada 24 horas, durante cinco dias. Os animais foram submetidos à eutanásia no final de cada momento e para tal procedimento foi utilizado pentobarbital na dose de $150 \mathrm{mg} / \mathrm{kg}$ por via intraperitoneal. Foi colhida amostra sanguínea, uma única vez, por punção intracardíaca.

Foram realizados hemograma, contagem de plaquetas e bioquímica sérica (ureia, creatinina, aspartato aminotransferse- AST, fosfatase alcalina - FA, gamaglutamiltransferase - GGT, proteínas total - PT sérica, albumina, globulina, sódio e potássio). Foi realizada a diluição manual em câmara hematimétrica para as contagens das hemácias, leucócitos e plaquetas e realizado diferencial em 100 leucócitos. Para a dosagem da hemoglobina, foi utilizado o método da cianometahemoglobina por meio do aparelho Celm SB-190. A mensuração da PT plasmática foi realizada por refratometria e o hematócrito pelo método do microhematócrito. Para as dosagens dos exames bioquímicos foram utilizados kits comerciais da $\mathrm{Katal}^{\circledR}$ (ureia, AST, FA, GGT, PT, albumina), Laborlab ${ }^{\circledR}$ (creatinina) e Celm (sódio e potássio). Foram utilizados métodos de espectofotometria (colorimétrico) nas dosagens de ureia, PT sérica e albumina e método cinético nas dosagens de AST, FA, GGT e creatinina, para ambos os métodos utilizou-se o aparelho Celm SB-190 e com atividade enzimática a $37^{\circ} \mathrm{C}$. Para as dosagens de sódio e potássio, o método de fotometria de chama por meio do aparelho FC-280 foi aplicado.

Foi feita a análise de variância (ANOVA) de dois fatores, seguido do teste de Tukey para comparações múltiplas. O programa utilizado foi o SAS, versão 9.1.3.

\section{Resultados e Discussão}

Como o presente trabalho é experimental, os resultados serão exclusivamente comparados com trabalhos realizados em ratos. Não há, na literatura, dados sobre hemograma e exames bioquímicos em ratos com o uso do AINE firocoxibe para que se possa compará-los com os do presente trabalho. O uso dos AINES meloxicam e firocoxibe não causou alterações significativas nos parâmetros hematológicos e bioquímicos (Tabelas 1, 2, 3 e 4). As alterações mais significativas ocorreram com os animais do grupo G2 (Tabelas 1, 2, 3 e 4).

$\mathrm{Na}$ hematimetria, existiu diferença significativa, entre as médias dos grupos, das hemácias, hematócrito e hemoglobina. Sendo que o grupo G2 apresentou a menor média em comparação aos demais grupos (Tabela 3). Houve diferença significativa também entre os diferentes momentos e grupos de avaliação, em relação à hemoglobina. Nos momentos M1 e M2, os valores médios da hemoglobina do G2 foram menores em comparação aos outros grupos. No G2, os valores médios da hemoglobina foram maiores no M3 em comparação aos momentos M1 e M2 (Tabela 1). Estes dados estão de acordo com alterações sugestivas de anemia encontradas em ratos Wistar que receberam diclofenaco $(3 \mathrm{mg} / \mathrm{kg}$, a cada 12 horas, por via intragástrica, 14 dias), possivelmente relacionadas ao sangramento gastrointestinal após administração do diclofenaco, sendo consistente com a injúria intestinal observada macroscopicamente neste trabalho ${ }^{19}$.

Não houve alterações significativas das plaquetas (Tabelas 1 e 3). Este fato também foi observado em ratos Wistar tratados com meloxicam $(3,75 \mathrm{mg} / \mathrm{kg}$, a cada 24 horas, por via intragástrica durante 14 e 28 dias) ${ }^{10}$. Por outro lado, podem ocorrer trombocitopenia com o uso de AINES $^{1}$, contrariando os dados obtidos neste trabalho. Além disso, foram observadas elevações na contagem de plaquetas em ratos Wistar que receberam diclofenaco durante 14 dias $^{19}$. A trombocitose pode ter ocorrido secundariamente a hemorragias crônicas ${ }^{20}$.

Outras alterações hematológicas que podem ocorrer com o uso de AINES são leucopenias e agranulo- 
Tabela 1 - Valores médios do Hemograma segundo grupos e momentos de avaliação - Botucatu - 2008

\begin{tabular}{|c|c|c|c|c|c|c|}
\hline & G1 & G2 & G3 & G4 & G5 & G6 \\
\hline \multicolumn{7}{|c|}{ Hemácias $10^{6} / \mu \mathrm{L}(\mathrm{p}=0,5780)$} \\
\hline M1 & $5,74 \mathrm{Aa}$ & $4,84 \mathrm{Aa}$ & $6,11 \mathrm{Aa}$ & $5,91 \mathrm{Aa}$ & $6,19 \mathrm{Aa}$ & $6,36 \mathrm{Aa}$ \\
\hline M2 & $5,11 \mathrm{Aa}$ & $4,08 \mathrm{Aa}$ & $4,56 \mathrm{Aa}$ & $5,12 \mathrm{Aa}$ & $5,23 \mathrm{Aa}$ & $5,03 \mathrm{Aa}$ \\
\hline M3 & $5,41 \mathrm{Aa}$ & 4,55 Aa & $5,82 \mathrm{Aa}$ & $5,56 \mathrm{Aa}$ & $5,19 \mathrm{Aa}$ & $5,06 \mathrm{Aa}$ \\
\hline \multicolumn{7}{|c|}{ Hemoglobina $\mathrm{g} / \mathrm{dL}(\mathrm{p}=0,0432)$. } \\
\hline M1 & $12,67 \mathrm{Aa}$ & $9,84 \mathrm{Ab}$ & $12,14 \mathrm{Aa}$ & $11,58 \mathrm{Aa}$ & $11,94 \mathrm{Aa}$ & $12,40 \mathrm{Aa}$ \\
\hline M2 & $12,97 \mathrm{Aa}$ & $10,22 \mathrm{Ab}$ & $12,38 \mathrm{Aa}$ & $12,48 \mathrm{Aa}$ & $12,94 \mathrm{Aa}$ & $12,60 \mathrm{Aa}$ \\
\hline M3 & $12,16 \mathrm{Aa}$ & $11,84 \mathrm{Ba}$ & $12,24 \mathrm{Aa}$ & $11,82 \mathrm{Aa}$ & $12,34 \mathrm{Aa}$ & $12,44 \mathrm{Aa}$ \\
\hline \multicolumn{7}{|c|}{ Hematócrito \% $(p=0,1748)$} \\
\hline M1 & $42,50 \mathrm{Aa}$ & $35,60 \mathrm{Aa}$ & $40,80 \mathrm{Aa}$ & $40,80 \mathrm{Aa}$ & $39,80 \mathrm{Aa}$ & $43,60 \mathrm{Aa}$ \\
\hline M2 & $43,50 \mathrm{Aa}$ & $34,20 \mathrm{Aa}$ & $42,20 \mathrm{Aa}$ & $42,20 \mathrm{Aa}$ & $44,20 \mathrm{Aa}$ & $43,20 \mathrm{Aa}$ \\
\hline M3 & $42,40 \mathrm{Aa}$ & $39,29 \mathrm{Aa}$ & $40,20 \mathrm{Aa}$ & $40,20 \mathrm{Aa}$ & $42,00 \mathrm{Aa}$ & $43,20 \mathrm{Aa}$ \\
\hline \multicolumn{7}{|c|}{ PT plasmática $\mathrm{g} / \mathrm{dL}(\mathrm{p}=0,8548)$. } \\
\hline M1 & $6,00 \mathrm{Aa}$ & $5,48 \mathrm{Aa}$ & $5,68 \mathrm{Aa}$ & $5,64 \mathrm{Aa}$ & $5,96 \mathrm{Aa}$ & $5,84 \mathrm{Aa}$ \\
\hline M2 & $6,03 \mathrm{Aa}$ & $5,56 \mathrm{Aa}$ & $6,08 \mathrm{Aa}$ & $6,2 \mathrm{Aa}$ & $6,16 \mathrm{Aa}$ & $6,32 \mathrm{Aa}$ \\
\hline M3 & $6,52 \mathrm{Aa}$ & $5,89 \mathrm{Aa}$ & $6,16 \mathrm{Aa}$ & $6,32 \mathrm{Aa}$ & $6,28 \mathrm{Aa}$ & $6,4 \mathrm{Aa}$ \\
\hline \multicolumn{7}{|c|}{ Plaquetas $10^{5} / \mu \mathrm{L}(\mathrm{p}=0,4635)$} \\
\hline M1 & $6,28 \mathrm{Aa}$ & $5,14 \mathrm{Aa}$ & 7,38 Aa & $6,62 \mathrm{Aa}$ & $5,63 \mathrm{Aa}$ & $6,23 \mathrm{Aa}$ \\
\hline M2 & $6,24 \pm 1,32$ & $6,22 \mathrm{Aa}$ & $6,36 \mathrm{Aa}$ & $6,87 \mathrm{Aa}$ & $6,44 \mathrm{Aa}$ & $6,10 \mathrm{Aa}$ \\
\hline M3 & 6,74 Aa & 7,63 Aa & $6,45 \mathrm{Aa}$ & $6,94 \mathrm{Aa}$ & $6,70 \mathrm{Aa}$ & $7,28 \mathrm{Aa}$ \\
\hline \multicolumn{7}{|c|}{ Leucócitos $10^{3} / \mu \mathrm{L}(\mathrm{p}=0,2594)$} \\
\hline M1 & 6,89 Aa & 7,52 Aa & 7,34 Aa & $6,52 \mathrm{Aa}$ & $5,54 \mathrm{Aa}$ & $6,06 \mathrm{Aa}$ \\
\hline M2 & $7,18 \mathrm{Aa}$ & $6,77 \mathrm{Aa}$ & $6,26 \mathrm{Aa}$ & $8,19 \mathrm{Aa}$ & $7,72 \mathrm{Aa}$ & $5,81 \mathrm{Aa}$ \\
\hline M3 & $6,39 \mathrm{Aa}$ & $8,44 \mathrm{Aa}$ & $7,58 \mathrm{Aa}$ & $7,52 \mathrm{Aa}$ & $6,02 \mathrm{Aa}$ & $5,58 \mathrm{Aa}$ \\
\hline \multicolumn{7}{|c|}{ Neutrófilos $10^{3} / \mu \mathrm{L}(\mathrm{p}=0,4932)$} \\
\hline M1 & $1,73 \mathrm{Aa}$ & $2,06 \mathrm{Aa}$ & $1,95 \mathrm{Aa}$ & $1,92 \mathrm{Aa}$ & $1,95 \mathrm{Aa}$ & $2,04 \mathrm{Aa}$ \\
\hline M2 & $1,91 \mathrm{Aa}$ & $2,42 \mathrm{Aa}$ & $2,19 \mathrm{Aa}$ & $2,61 \mathrm{Aa}$ & $2,37 \mathrm{Aa}$ & $1,63 \mathrm{Aa}$ \\
\hline M3 & $1,68 \mathrm{Aa}$ & $2,71 \pm \mathrm{Aa}$ & $2,94 \mathrm{Aa}$ & $3,22 \mathrm{Aa}$ & $1,85 \mathrm{Aa}$ & $2,48 \mathrm{Aa}$ \\
\hline \multicolumn{7}{|c|}{ Linfócitos $10^{3} / \mu \mathrm{L}(\mathrm{p}=0,2074)$} \\
\hline M1 & 4,60 Aa & 4,72 Aa & $5,02 \mathrm{Aa}$ & 3,99 Aa & 3,33 Aa & $3,65 \mathrm{Aa}$ \\
\hline M2 & $4,90 \mathrm{Aa}$ & 4,47 Aa & $3,86 \mathrm{Aa}$ & $5,16 \mathrm{Aa}$ & $5,08 \mathrm{Aa}$ & $3,88 \mathrm{Aa}$ \\
\hline M3 & $4,39 \mathrm{Aa}$ & $5,21 \mathrm{Aa}$ & $4,08 \mathrm{Aa}$ & $3,78 \mathrm{Aa}$ & $3,84 \mathrm{Aa}$ & $3,40 \mathrm{Aa}$ \\
\hline \multicolumn{7}{|c|}{ Eosinófilos $10^{3} / \mu \mathrm{L}(\mathrm{p}=0,0586)$} \\
\hline M1 & $0,16 \mathrm{Aa}$ & $0,36 \mathrm{Aa}$ & $0,05 \mathrm{Aa}$ & $0,07 \mathrm{Aa}$ & $0,08 \mathrm{Aa}$ & $0,12 \mathrm{Aa}$ \\
\hline M2 & $0,13 \mathrm{Aa}$ & $0,13 \mathrm{Aa}$ & $0,10 \mathrm{Aa}$ & $0,04 \mathrm{Aa}$ & $0,13 \mathrm{Aa}$ & $0,09 \mathrm{Aa}$ \\
\hline M3 & $0,76 \mathrm{Aa}$ & $0,15 \mathrm{Aa}$ & $0,23 \mathrm{Aa}$ & $0,16 \mathrm{Aa}$ & $0,04 \mathrm{Aa}$ & $0,14 \mathrm{Aa}$ \\
\hline \multicolumn{7}{|c|}{ Basófilos $10^{3} / \mu \mathrm{L}(\mathrm{p}=0,0737)$} \\
\hline M1 & $0,02 \mathrm{Aa}$ & 0,05 Aa & $0,01 \mathrm{Aa}$ & $0,040 \mathrm{Aa}$ & $0,00 \mathrm{Aa}$ & $0,00 \mathrm{Aa}$ \\
\hline M2 & $0,01 \mathrm{Aa}$ & $0,00 \mathrm{Aa}$ & $0,01 \mathrm{Aa}$ & $0,01 \mathrm{Aa}$ & $0,00 \mathrm{Aa}$ & $0,09 \mathrm{Aa}$ \\
\hline M3 & $0,00 \mathrm{Aa}$ & $0,10 \mathrm{Aa}$ & $0,00 \mathrm{Aa}$ & $0,03 \mathrm{Aa}$ & $0,01 \mathrm{Aa}$ & $0,02 \mathrm{Aa}$ \\
\hline \multicolumn{7}{|c|}{ Monócitos $10^{3} / \mu \mathrm{L}(\mathrm{p}=0,4162)$} \\
\hline M1 & $0,34 \mathrm{Aa}$ & $0,42 \mathrm{Aa}$ & $0,28 \mathrm{Aa}$ & 0,40 Aa & $0,16 \mathrm{Aa}$ & $0,23 \mathrm{Aa}$ \\
\hline M2 & $0,21 \mathrm{Aa}$ & $0,49 \mathrm{Aa}$ & $0,08 \mathrm{Aa}$ & $0,35 \mathrm{Aa}$ & $0,13 \mathrm{Aa}$ & $0,19 \mathrm{Aa}$ \\
\hline M3 & $0,23 \mathrm{Aa}$ & $0,25 \mathrm{Aa}$ & $0,31 \mathrm{Aa}$ & $0,31 \mathrm{Aa}$ & $0,26 \mathrm{Aa}$ & $0,24 \mathrm{Aa}$ \\
\hline \multicolumn{7}{|c|}{ Bastonetes $10^{3} / \mu \mathrm{L}(\mathrm{p}=0,6602)$} \\
\hline M1 & $0,01 \mathrm{Aa}$ & $0,00 \mathrm{Aa}$ & $0,01 \mathrm{Aa}$ & $0,04 \mathrm{Aa}$ & $0,00 \mathrm{Aa}$ & $0,00 \mathrm{Aa}$ \\
\hline M2 & $0,00 \mathrm{Aa}$ & $0,00 \mathrm{Aa}$ & $0,00 \mathrm{Aa}$ & $0,00 \mathrm{Aa}$ & $0,00 \mathrm{Aa}$ & $0,00 \mathrm{Aa}$ \\
\hline M3 & $0,00 \mathrm{Aa}$ & $0,00 \mathrm{Aa}$ & $0,00 \mathrm{Aa}$ & $0,00 \mathrm{Aa}$ & $0,00 \mathrm{Aa}$ & $0,00 \mathrm{Aa}$ \\
\hline
\end{tabular}

G1: controle 0,3 mL/100 g de p.v. de S. F.; G2: diclofenaco de sódio $15 \mathrm{mg} / \mathrm{kg}$; G3: meloxicam 2,0 mg/kg;

G4: meloxicam $10 \mathrm{mg} / \mathrm{kg}$; G5: firocoxibe $5,0 \mathrm{mg} / \mathrm{kg}$; G6: firocoxibe: $25,0 \mathrm{mg} / \mathrm{kg}$ ).

M1: $48 \mathrm{~h}$ após o início do tratamento; M2: $96 \mathrm{~h}$ após o início do tratamento; M3: $72 \mathrm{~h}$ após o término do tratamento

$\mathrm{n}=5$ animais para todos os grupos em cada momento de avaliação, com exceção do G1 em M1 e M2 (n= 6) e G2 em M3 (n = 7).

Letras maiúsculas: comparação entre momentos (linhas); letras minúsculas: comparação entre grupos (colunas); letras iguais não diferem entre si; $\mathrm{p}<0,05)$ 
Tabela 2 - Valores médios dos Exames Bioquímicos segundo grupos e momentos de avaliação - Botucatu - 2008

\begin{tabular}{|c|c|c|c|c|c|c|}
\hline & G1 & G2 & G3 & G4 & G5 & G6 \\
\hline \multicolumn{7}{|c|}{ AST UI/L $(p=0,8201)$} \\
\hline M1 & $93,83 \mathrm{Aa}$ & $79,6 \mathrm{Aa}$ & $85,2 \mathrm{Aa}$ & $87,6 \mathrm{Aa}$ & $80,2 \mathrm{Aa}$ & $80,4 \mathrm{Aa}$ \\
\hline M2 & $92,5 \mathrm{Aa}$ & $90,4 \mathrm{Aa}$ & $94,4 \mathrm{Aa}$ & $87,4 \mathrm{Aa}$ & $96 \mathrm{Aa}$ & $84,8 \mathrm{Aa}$ \\
\hline M3 & $86,2 \mathrm{Aa}$ & $79,86 \mathrm{Aa}$ & $86,6 \mathrm{Aa}$ & $79,8 \mathrm{Aa}$ & $82,4 \mathrm{Aa}$ & $82,6 \mathrm{Aa}$ \\
\hline \multicolumn{7}{|c|}{ FA UI/dL $(p=0,7781)$} \\
\hline M1 & $60,73 \mathrm{Aa}$ & $48,77 \mathrm{Aa}$ & $61,67 \mathrm{Aa}$ & $52,53 \mathrm{Aa}$ & $58,10 \mathrm{Aa}$ & $57,71 \mathrm{Aa}$ \\
\hline M2 & $55,72 \mathrm{Aa}$ & $54,65 \mathrm{Aa}$ & 64,92 Aa & $59,69 \mathrm{Aa}$ & $56,32 \mathrm{Aa}$ & $52,50 \mathrm{Aa}$ \\
\hline M3 & $52,95 \mathrm{Aa}$ & $53,29 \mathrm{Aa}$ & $57,56 \mathrm{Aa}$ & $67,94 \mathrm{Aa}$ & $66,45 \mathrm{Aa}$ & $59,37 \mathrm{Aa}$ \\
\hline \multicolumn{7}{|c|}{ GGT UI/L (p = 0,6688) } \\
\hline M1 & $1,28 \mathrm{Aa}$ & $1,82 \pm 0,80$ & $1,12 \pm 0,38$ & $1,12 \pm 0,63$ & $1,12 \pm 0,63$ & $1,54 \pm 0,91$ \\
\hline M2 & $1,52 \mathrm{Aa}$ & $1,4 \pm 0,70$ & $1,4 \pm 0,50$ & $1,4 \pm 0,86$ & $0,98 \pm 0,38$ & $1,4 \pm 0,50$ \\
\hline M3 & $1,12 \mathrm{Aa}$ & $1,1 \pm 0,55$ & $1,4 \pm 0,99$ & $1,4 \pm 0,50$ & $1,4 \pm 0,86$ & $0,98 \pm 0,38$ \\
\hline \multicolumn{7}{|c|}{$\mathrm{PT}$ sérica $\mathrm{g} / \mathrm{dL}(\mathrm{p}=0,8609)$} \\
\hline M1 & $5,63 \pm 0,43$ & 4,4 Aa & $5,04 \mathrm{Aa}$ & $5,3 \mathrm{Aa}$ & $5,4 \mathrm{Aa}$ & $5,36 \mathrm{Aa}$ \\
\hline M2 & $5,5 \pm 0,22$ & $4,96 \mathrm{Aa}$ & $5,48 \mathrm{Aa}$ & $5,52 \mathrm{Aa}$ & 5,56 Aa & $5,68 \mathrm{Aa}$ \\
\hline M3 & $5,9 \pm 0,28$ & $5,00 \mathrm{Aa}$ & $5,56 \mathrm{Aa}$ & $5,68 \mathrm{Aa}$ & $5,72 \mathrm{Aa}$ & $5,84 \mathrm{Aa}$ \\
\hline \multicolumn{7}{|c|}{ Albumina $\mathrm{g} / \mathrm{dL}(\mathrm{p}=\mathbf{0 , 4 3 3 8 )}$} \\
\hline M1 & $3,56 \mathrm{Aa}$ & $3,31 \mathrm{Aa}$ & $3,24 \mathrm{Aa}$ & $3,04 \mathrm{Aa}$ & $3,18 \mathrm{Aa}$ & $3,3 \mathrm{Aa}$ \\
\hline M2 & $3,2 \mathrm{Aa}$ & $2,79 \mathrm{Aa}$ & $3,06 \mathrm{Aa}$ & $3,00 \mathrm{Aa}$ & $3,25 \mathrm{Aa}$ & 3,3 Aa \\
\hline M3 & $3,66 \mathrm{Aa}$ & $3,17 \mathrm{Aa}$ & $3,27 \mathrm{Aa}$ & $3,51 \mathrm{Aa}$ & $3,44 \mathrm{Aa}$ & $3,35 \mathrm{Aa}$ \\
\hline \multicolumn{7}{|c|}{ Globulina $\mathrm{g} / \mathrm{dL}(\mathrm{p}=0,4929)$} \\
\hline M1 & $2,07 \mathrm{Aa}$ & $1,08 \mathrm{Aa}$ & $1,8 \mathrm{Aa}$ & $2,26 \mathrm{Aa}$ & $2,22 \mathrm{Aa}$ & $2,06 \mathrm{Aa}$ \\
\hline M2 & $2,3 \mathrm{Aa}$ & $2,16 \mathrm{Aa}$ & $2,42 \mathrm{Aa}$ & $2,52 \mathrm{Aa}$ & $2,31 \mathrm{Aa}$ & $2,37 \mathrm{Aa}$ \\
\hline M3 & $2,24 \mathrm{Aa}$ & $1,82 \mathrm{Aa}$ & $2,29 \mathrm{Aa}$ & $2,17 \mathrm{Aa}$ & $2,27 \mathrm{Aa}$ & $2,49 \mathrm{Aa}$ \\
\hline \multicolumn{7}{|c|}{ Ureia mg/dL ( $p=0,0337)$} \\
\hline M1 & $43,77 \mathrm{Aab}$ & $46,32 \mathrm{Aa}$ & $41,2 \mathrm{Aab}$ & $44,44 \mathrm{Aab}$ & $39,06 \mathrm{Ab}$ & $39,00 \mathrm{Ab}$ \\
\hline M2 & $41,85 \mathrm{Aa}$ & $39,72 \mathrm{Ba}$ & $45,16 \mathrm{ABa}$ & $40,56 \mathrm{Aa}$ & $45,74 \mathrm{Ba}$ & $44,36 \mathrm{Aa}$ \\
\hline M3 & $42,36 \mathrm{Aa}$ & $41,59 \mathrm{ABa}$ & $47,68 \mathrm{Ba}$ & $41,36 \mathrm{Aa}$ & $41,72 \mathrm{ABa}$ & $41,98 \mathrm{Aa}$ \\
\hline \multicolumn{7}{|c|}{ Creatinina $\mathrm{mg} / \mathrm{dL}(\mathrm{p}=0,4484)$} \\
\hline M1 & $0,48 \mathrm{Aa}$ & $0,42 \mathrm{Aa}$ & $0,5 \mathrm{Aa}$ & $0,52 \mathrm{Aa}$ & $0,5 \mathrm{Aa}$ & $0,44 \mathrm{Aa}$ \\
\hline M2 & $0,45 \mathrm{Aa}$ & $0,48 \mathrm{Aa}$ & $0,48 \mathrm{Aa}$ & $0,44 \pm 0,05$ & $0,48 \mathrm{Aa}$ & $0,48 \mathrm{Aa}$ \\
\hline M3 & $0,52 \mathrm{Aa}$ & $0,44 \mathrm{Aa}$ & $0,58 \mathrm{Aa}$ & $0,5 \mathrm{Aa}$ & $0,52 \mathrm{Aa}$ & $0,5 \mathrm{Aa}$ \\
\hline \multicolumn{7}{|c|}{ Sódio $\mathrm{mEq} / \mathrm{L}(\mathrm{p}=\mathbf{0 , 0 3 5 1 )}$} \\
\hline M1 & $151,50 \mathrm{Aa}$ & $162,40 \mathrm{Aa}$ & $147,80 \mathrm{Ab}$ & $147,80 \mathrm{Ab}$ & $145,20 \mathrm{Ab}$ & $145,80 \mathrm{Ab}$ \\
\hline M2 & $142,17 \mathrm{Aa}$ & $139,60 \mathrm{Ba}$ & $145,20 \mathrm{Aa}$ & $141,40 \mathrm{Aa}$ & $147,60 \mathrm{Aa}$ & $146,00 \mathrm{Aa}$ \\
\hline M3 & $145,2 \mathrm{Aa}$ & $156,14 \mathrm{Aa}$ & $148,20 \mathrm{Aa}$ & $146,60 \mathrm{Aa}$ & $145,00 \mathrm{Aa}$ & $145,00 \mathrm{Aa}$ \\
\hline \multicolumn{7}{|c|}{ Potássio $\mathrm{mEq} / \mathrm{L}(\mathrm{p}=\mathbf{0 , 1 5 6 4 )}$} \\
\hline M1 & $5,60 \mathrm{Aa}$ & $6,28 \mathrm{Aa}$ & $5,3 \mathrm{Aa}$ & $5,64 \mathrm{Aa}$ & 4,94 Aa & $5,32 \mathrm{Aa}$ \\
\hline M2 & $5,50 \mathrm{Aa}$ & $5,86 \mathrm{Aa}$ & $5,48 \mathrm{Aa}$ & $5,46 \mathrm{Aa}$ & $6,14 \mathrm{Aa}$ & $5,46 \mathrm{Aa}$ \\
\hline M3 & $5,12 \mathrm{Aa}$ & $5,81 \mathrm{Aa}$ & $6,02 \mathrm{Aa}$ & $5,28 \mathrm{Aa}$ & $5,62 \mathrm{Aa}$ & $5,46 \mathrm{Aa}$ \\
\hline
\end{tabular}

G1: controle 0,3 mL/100 g de p.v. de S. F.; G2: diclofenaco de sódio $15 \mathrm{mg} / \mathrm{kg}$; G3: meloxicam 2,0 mg/kg;

G4: meloxicam $10 \mathrm{mg} / \mathrm{kg}$; G5: firocoxibe $5,0 \mathrm{mg} / \mathrm{kg}$; G6: firocoxibe: $25,0 \mathrm{mg} / \mathrm{kg}$.

M1: 48 h após o início do tratamento; M2: $96 \mathrm{~h}$ após o início do tratamento; M3: $72 \mathrm{~h}$ após o término do tratamento

$\mathrm{N}=5$ animais para todos os grupos em cada momento de avaliação, com exceção do G1 em M1 e M2 (n = 6) e G2 em M3 (n = 7).

Letras maiúsculas: comparação ente momentos (linhas); letras minúsculas: comparação entre grupos (colunas); letras iguais não diferem entre si; $\mathrm{p}<0,0$

citoses $^{1}$. No entanto, não houve diferença significativa nas contagens de leucócitos, bastonetes, neutrófilos, linfócitos, eosinófilos, basófilos e monócitos entre os diferentes momentos de avaliação e grupos (Tabela 1). Porém, existiu diferença significativa entre as médias dos grupos, sendo que o grupo G2 apresentou valores médios maiores de leucócitos em comparação aos do G6 e valores maiores de monócitos em comparação aos dos grupos G3, G5 e G6, mas sem diferenças significativas em comparação aos do grupo controle 
Tabela 3 - Valores médios dos grupos no Hemograma e Exames Bioquímicos - Botucatu - 2008

\begin{tabular}{|c|c|c|c|c|c|c|c|}
\hline & G1 & G2 & G3 & G4 & G5 & G6 & p \\
\hline \multicolumn{8}{|c|}{ Hemograma } \\
\hline Hemácias $10^{6} / \mu \mathrm{L}$ & $5,42 \mathrm{~A}$ & $4,49 \mathrm{~B}$ & $5,50 \mathrm{~A}$ & $5,53 \mathrm{~A}$ & $5,54 \mathrm{~A}$ & $5,48 \mathrm{~A}$ & 0,0004 \\
\hline Hemoglobina g/dL & $12,59 \mathrm{~A}$ & $10,63 \mathrm{~B}$ & $12.25 \mathrm{~A}$ & $11,96 \mathrm{~A}$ & $12,40 \mathrm{~A}$ & $12,48 \mathrm{~A}$ & $<0,0001$ \\
\hline Hematócrito \% & $42,80 \mathrm{~A}$ & $36,36 \mathrm{~B}$ & $41,93 \mathrm{~A}$ & $41,06 \mathrm{~A}$ & $42,00 \mathrm{~A}$ & $43,33 \mathrm{~A}$ & $<0,0001$ \\
\hline PT plasmática g/dL & $6,18 \mathrm{~A}$ & $5,64 \mathrm{~B}$ & $5,97 \mathrm{~A}$ & $6,05 \mathrm{~A}$ & $6,13 \mathrm{~A}$ & $6,18 \mathrm{~A}$ & 0,0020 \\
\hline Plaquetas $10^{5} / \mu \mathrm{L}$ & $6,42 \mathrm{~A}$ & $6,33 \mathrm{~A}$ & $6,73 \mathrm{~A}$ & $6,81 \mathrm{~A}$ & $6,26 \mathrm{~A}$ & $6,54 \mathrm{~A}$ & 0,8953 \\
\hline Leucócitos $10^{3} / \mu \mathrm{L}$ & $6,82 \mathrm{AB}$ & $7,58 \mathrm{~A}$ & $7,06 \mathrm{AB}$ & $7,41 \mathrm{AB}$ & $6,43 \mathrm{AB}$ & $5,82 \mathrm{~B}$ & 0,0261 \\
\hline Linfócitos $10^{3} / \mu \mathrm{L}$ & $4,63 \mathrm{~A}$ & $4,80 \mathrm{~A}$ & $4,32 \mathrm{~A}$ & $4,31 \mathrm{~A}$ & $4,09 \mathrm{~A}$ & $3,64 \mathrm{~A}$ & 0,0583 \\
\hline Eosinófilos $10^{3} / \mu \mathrm{L}$ & $0,12 \mathrm{~A}$ & $0,21 \mathrm{~A}$ & $0,13 \mathrm{~A}$ & $0,09 \mathrm{~A}$ & $0,08 \mathrm{~A}$ & $0,12 \mathrm{~A}$ & 0,1331 \\
\hline Basófilos $10^{3} / \mu \mathrm{L}$ & $0,01 \mathrm{~A}$ & $0,05 \mathrm{~B}$ & $0,00 \mathrm{~A}$ & $0,02 \mathrm{AB}$ & $0,00 \mathrm{~A}$ & $0,01 \mathrm{~B}$ & 0,0017 \\
\hline Monócitos $10^{3} / \mu \mathrm{L}$ & $0,26 \mathrm{ABC}$ & $0,39 \mathrm{~A}$ & $0,22 \mathrm{BC}$ & $0,35 \mathrm{AB}$ & $0,18 \mathrm{C}$ & $0,22 \mathrm{BC}$ & 0,0367 \\
\hline Bastonetes $10^{3} / \mu \mathrm{L}$ & $0,00 \mathrm{~A}$ & $0,00 \mathrm{~A}$ & $0,00 \mathrm{~A}$ & $0,01 \mathrm{~A}$ & $0,00 \mathrm{~A}$ & $0,00 \mathrm{~A}$ & 0,5718 \\
\hline \multicolumn{8}{|c|}{ Exames bioquímicos } \\
\hline AST UI/L & $90,84 \mathrm{~A}$ & $83,28 \mathrm{~A}$ & $88,73 \mathrm{~A}$ & $84,93 \mathrm{~A}$ & $86,20 \mathrm{~A}$ & $82,60 \mathrm{~A}$ & 0,2039 \\
\hline FA UI/dL & $564,72 \mathrm{~A}$ & $522,44 \mathrm{~A}$ & $613,84 \mathrm{~A}$ & $600,58 \mathrm{~A}$ & $602,95 \mathrm{~A}$ & $565,29 \mathrm{~A}$ & 0,4837 \\
\hline $\mathrm{PT}$ sérica $\mathrm{g} / \mathrm{dL}$ & $5,67 \mathrm{~A}$ & $4,78 \mathrm{~B}$ & $5,36 \mathrm{~A}$ & $5,50 \mathrm{~A}$ & $5,56 \mathrm{~A}$ & $5,62 \mathrm{~A}$ & $<0,0001$ \\
\hline Albumina g/dL & $3,47 \mathrm{~A}$ & $3,09 \mathrm{~B}$ & $3,19 \mathrm{AB}$ & $3,18 \mathrm{AB}$ & $3,29 \mathrm{AB}$ & $3,31 \mathrm{AB}$ & 0,0365 \\
\hline Globulina g/dL & $2,20 \mathrm{~A}$ & $1,69 \mathrm{~B}$ & $2,16 \mathrm{AB}$ & $2,31 \mathrm{~A}$ & $2,26 \mathrm{~A}$ & $2,30 \mathrm{~A}$ & 0,0041 \\
\hline Ureia mg/dL & $42,65 \mathrm{~A}$ & $42,54 \mathrm{~A}$ & $44,68 \mathrm{~A}$ & $42,12 \mathrm{~A}$ & $42,17 \mathrm{~A}$ & $41,78 \mathrm{~A}$ & 0,5880 \\
\hline Creatinina $\mathrm{mg} / \mathrm{dL}$ & $\begin{array}{c}0,48 \mathrm{~A} \\
14628 \mathrm{AB}\end{array}$ & $0,44 \mathrm{~A}$ & $0,52 \mathrm{~A}$ & $0,48 \mathrm{~A}$ & $0,50 \mathrm{~A}$ & $0,47 \mathrm{~A}$ & 0,1126 \\
\hline Sódio $\mathrm{mEq} / \mathrm{L}$ & $\begin{array}{c}146,28 \mathrm{AB} \\
5,40 \mathrm{~A}\end{array}$ & $152,71 \mathrm{~B}$ & $147,06 \mathrm{AB}$ & $145,26 \mathrm{~A}$ & $145,93 \mathrm{AB}$ & $145,60 \mathrm{AB}$ & 0,0319 \\
\hline Potássio $\mathrm{mEq} / \mathrm{L}$ & $5,40 \mathrm{~A}$ & $5,98 \mathrm{~A}$ & $5,60 \mathrm{~A}$ & $5,46 \mathrm{~A}$ & $5,56 \mathrm{~A}$ & $5,41 \mathrm{~A}$ & 0,1586 \\
\hline
\end{tabular}

G1: controle 0,3 mL/100 g de p.v. de S. F.; G2: diclofenaco de sódio 15 mg/kg; G3: meloxicam 2,0 mg/kg; G4: meloxicam 10 mg/kg; G5: firocoxibe 5,0 $\mathrm{mg} / \mathrm{kg}$; G6: firocoxibe: $25,0 \mathrm{mg} / \mathrm{kg}$.

$\mathrm{N}=17$ animais para os grupos G1 e G2 e $\mathrm{n}=15$ animais para os grupos G3, G4, G5 e G6.

Letras maiúsculas: comparação entre grupos (linhas)

Tabela 4 - Valores médios dos momentos no Hemograma e Exames Bioquímicos - Botucatu - 2008

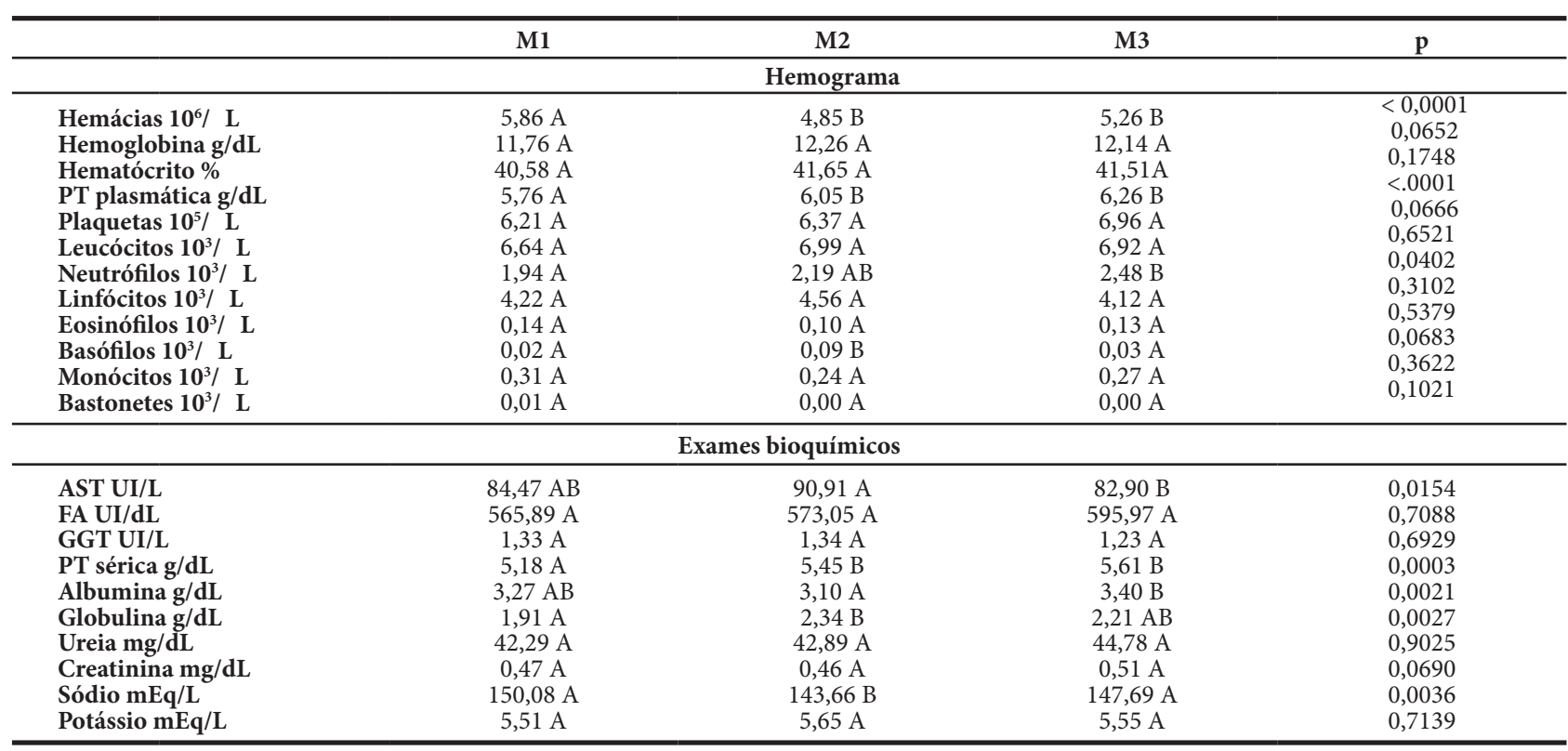

M1: 48 h após o início do tratamento; M2: 96 h após o início do tratamento; M3: 72 h após o término do tratamento

$\mathrm{n}=31$ animais para os momentos M1 e M2 e $\mathrm{n}=32$ animais para o momento M3.

Letras maiúsculas: comparação entre momentos (linhas)

(Tabela 3). Não se observaram também as alterações na contagem de leucócitos em ratos Wistar tratados com diclofenaco ${ }^{19}$ e meloxicam ${ }^{10}$. No presente traba- lho, existiu diferença significativa da contagem dos basófilos entre as médias dos grupos, sendo que o G2 apresentou valores médios maiores em comparação 
aos dos grupos G1, G3, G5 e G6 (Tabela 3). A basofilia acompanha frequentemente a eosinofilia causada por inflamação crônica das superfícies mucosa e cutânea $^{20}$. No presente estudo, 35,29\% dos animais do G2 apresentaram diarreia, no entanto, não foi observada diferença significativa na contagem dos eosinófilos. Na literatura consultada, não há menção da basofilia com o uso de AINES.

Os AINES podem causar lesão hepática, provocada muito mais por mecanismo idiossincrático do que por efeito tóxico direto ${ }^{21}$. Sabe-se que as investigações bioquímicas da função hepática podem ajudar a diferenciar condições como obstrução do trato biliar, lesão hepatocelular aguda e doença crônica do fígado, produzidas ou não por drogas ${ }^{22}$. Elevações das transaminases são comumente associadas ao uso dos AINES, entretanto insuficiência hepática é muito rara ${ }^{23}$.

No presente estudo, não se observaram alterações significativas da AST, FA e GGT (Tabelas 2 e 3) estando de acordo com os dados em ratos Sprague- Dawley tratados com diclofenaco de sódio $(15 \mathrm{mg} / \mathrm{kg}$, a cada 24 horas, por via intragástrica, sete dias $)^{15}$. Contrapondo os resultados obtidos no presente estudo, foi observada redução da AST e elevada atividade sérica da GGT em ratos Wistar que receberam diclofenaco em um período maior de tratamento (14 dias), mas não se observaram alterações significativas da $\mathrm{FA}^{19}$. Em ratos Wistar tratados com meloxicam foi observado aumento significativo da AST aos 14 dias de tratamento, porém após os 14 dias, os valores retornaram à normalidade. Também não se observaram alterações significativas da FA e $\mathrm{GGT}^{10}$, estando de acordo com os resultados observados no presente estudo.

A proteína total sérica reflete uma combinação entre a albumina e as globulinas. O total de proteínas plasmáticas é maior do que o valor determinado de forma bioquímica no soro. A diferença representa a concentração de fibrinogênio utilizada durante a formação do coágulo ${ }^{24}$. Não houve diferença significativa das proteínas plasmática e total sérica, albumina e globulina entre os diferentes momentos de avaliação e grupos (Tabelas 1 e 2). Mas, existiu diferença significativa entre as médias dos grupos, sendo que o grupo G2 apresentou valores médios menores da proteína plasmática em comparação aos dos grupos G1, G5 e G6; valores menores da proteína total sérica em comparação aos dos outros grupos; valores menores de albumina em comparação aos do grupo G1 e valores menores de globulina em comparação aos dos grupos G1, G4, G5 e G6 (Tabela 3). Perdas de globulina podem ocorrer por hemorragias e pelos sistemas gastrointestinal e renal ${ }^{20}$. A albumina é produzida exclusivamente pelo fígado e as causas de hipoalbuminemia incluem insuficiência hepática, glomerulonefropatias, enteropatia com perdas de proteínas, secundariamente a hiperglobulinemia ${ }^{24,25}$ e inanição ${ }^{24}$. Valores diminuídos da albumina foram também observados em ratos SpragueDawley tratados com diclofenaco de sódio, como resultado da diminuição da síntese hepática ${ }^{15}$.

A toxicidade renal dos AINES deve-se à presença das COX-1 e COX-2 neste órgão ${ }^{2}$. Diminuição do fluxo sanguíneo renal e da filtração glomerular, insuficiência renal aguda, retenção de sódio e água, nefrite intersticial, necrose papilar e insuficiência renal crônica são efeitos produzidos pelos AINES pela diminuição da síntese das $\mathrm{PGs}^{22}$. A ureia e a creatinina são indicadoras de filtração glomerular e apenas a ureia é influenciada pela dieta ou por hemorragias intestinais ${ }^{24}$.

Não existiu diferença significativa entre as médias dos grupos em relação à ureia e à creatinina (Tabela 3 ). No entanto, houve diferença significativa da ureia entre os diferentes momentos de avaliação e grupos. No momento M1, o G2 apresentou valores maiores de ureia em comparação aos dos grupos G4 e G5, mas não apresentou diferenças significativas com o grupo controle (Tabela 2). Por outro lado, foram observadas elevações significativas da ureia em ratos Wistar que receberam diclofenaco durante 14 dias e, como não houve alterações significativas da creatinina, esta elevação da ureia foi atribuída à hemorragia gastrintestinal ${ }^{19}$. Não foram encontradas alterações significativas na ureia e na creatinina em ratos Wistar tratados com meloxicam ${ }^{10}$, concordando com os dados do presente estudo. 
De acordo com a literatura, os AINES possuem efeitos antinatriuréticos pela inibição das $\mathrm{PGs}^{25}$. Houve diferença significativa do sódio entre os diferentes momentos de avaliação e grupos. No momento M1, o G2 apresentou valores maiores de sódio em comparação aos dos grupos G3, G4, G5 e G6, mas não apresentou diferenças significativas com os do grupo controle (Tabela 2). Houve também diferenças significativas entre as médias dos grupos, sendo que o G2 apresentou valores maiores em comparação aos do G4, mas sem diferenças significativas com o grupo controle (Tabela 3). A hipercalemia pode ser resultante da redução na liberação de renina mediada por PG,

\section{Referências}

1.ANDRADE, S. F.; JERICÓ, M. M. Antiinflamatórios. In: ANDRADE, S. F. Manual de terapêutica veterinária. 2. ed. São Paulo: Roca, 2002. p. 89-113.

2.BRICKS, L. F.; SILVA, C. A. A. Toxicidade dos antiinflamatórios não-hormonais. Pediatria, v. 27, n. 3, p. 181-193, 2005.

3.LASCELlES, B. D. X.; COURT, M. H.; HARDIE, E. M.; ROBERTESON, S. A. Nonsteroidal anti-inflammatory drugs in cats: a review. Veterinary Anaesthesia and Analgesia, v. 34, n. 4, p. 228-250, 2007.

4.FRESNO, L.; MOLL, J.; PEÑALBA, B.; ESPADA, Y.; ANDALUZ, A.; PRANDI, D.; GOPEGUI, R. R.; GARCÍA, F. Effects of preoperative administration of meloxicam on whole blood platelet aggregation, buccal mucosal bleeding time, and haematological indices in dogs undergoing elective ovariohysterectomy. The Veterinary Journal, v. 170, p. 138-140, 2005.

5.BASSANEZI, B. S. B.; OLIVEIRA FILHO, A. G. Analgesia pós-operatória. Revista do Colégio Brasileiro de Cirurgiões, v. 33, n. 2, p. 116-122, 2006.

6.BOOTHE, D. M. Anti-inflammatory drugs. In: BOOTHE, D. M. Small animal clinical pharmacology and therapeutics. Philadelphia: Saunders, 2001. chap.16, p. 281-311.

7.POURJAFAR, M.; DERAKHSHANFAR, A. A histopathologic study on the side effects of the diclofenac sodium in rabbits. In: WORLD CONGRESS OF THE WORLD SMALL ANIMAL VETERINARY ASSOCIATION, 29., 2004, Rhodes, Grece. Proceedings... Disponível em: <http://www.vin.com. $>$. Acesso em: 07 fev. 2007

8. GLEED, R. D.; LUDDERS, J. W. The science and art of analgesia. In: RECENT ADVANCES IN VETERINARY ANESTHESIA AND ANALGESIA, 2006. Ithaca NY. Anais... Disponível em: $<$ http:// www.ivis.org.>. Acesso em: 14 set. 2007.

9.COSTA, P. R. S.; ARAÚJO, R. B.; COSTA, M. C.; MAIA, R. E. N. Endoscopia gastroduodenal após administração de nimesulida, monofenilbutazona e meloxicam em cães. Arquivo Brasileiro de Medicina Veterinária e Zootecnia, v. 59, n. 4, p. 903-909, 2007.

10.VILLEGAS, I.; ALARCON DE LA LASTRA, C.; MARTIN, M. J.; MOTILVA, V.; LA CASA GARCIA, C. Gastric damage que por sua vez promove uma redução na formação de aldosterona e decréscimo na excreção de potássio no túbulo distal ${ }^{26}$. Contrariamente aos dados encontrados na literatura, não foram observadas alterações significativas do potássio sérico em todos os grupos estudados, no presente trabalho (Tabelas 2 e 3).

\section{Conclusões}

Conclui-se que o diclofenaco de sódio não produz grandes alterações no hemograma e exames bioquímicos, enquanto que, o meloxicam e ofirocoxibe não produzem alterações e efeitos deletérios dose-dependentes nestes exames laboratoriais.

induced by subchronic administration of preferential cyclooxygenase- 1 and cyclooxygenase- 2 inhibitors in rats. Pharmacology, v. 66, n. 2, p. 68-75, 2002.

11.ALENCAR, M. M. A.; PINTO, M. T.; OLIVEIRA, D. M.; PESSOA, P. A. W.; CÂNDIDO, I. A.; VIRGÍNIO, C. G.; COELHO, H. S. M.; ROCHA, M. F. G. Margem de segurança do meloxicam em cães: efeitos deletérios nas células sangüíneas e trato gastrointestinal. Ciência Rural, v. 33, n. 3, p. 525-532, 2003.

12.HANSON, P. D.; ROMANO, D.; FLEISHMAN, C.; DRAG, M.; POLLMEIER, M.; GOGOLEWSKI, R.; TOULEMONDE, C.; ALVA, R. Health events recorded from 575 dogs treated for osteoarthritis with firocoxib, carprofen or etodolac. In: AMERICAN COLLEGE OF VETERINARY INTERNAL MEDICINE, 2004. Anais... Disponível em: < http://www.vin. com.>. Acesso em: 27 fev. 2007.

13. KVATERNICK, V.; POLLMEIER, M.; FISCHER, J.; HANSON, P. D. Pharmacokinetics and metabolism of orally administered firocoxib, a novel second generation coxib, in horses. Journal of Veterinary Pharmacology and Therapeutics, v. 30, n. 3, p. 208-217, 2007.

14.STEAGALL, P. V. M.; MANTOVANI, F. B.; FERREIRA, T. H.; SALCEDO. E. S.; MOUTINHO, F. Q.; LUNA, S. P. Evaluation of the adverse effects of oral firocoxib in healthy dogs. Journal of Veterinary Pharmacology and Therapeutics, v. 30, n. 3, p. 218-223, 2007.

15. SALLUSTIO, B. C.; HOLBROOK, F. L. In vivo pertubation of rat hepatocyte canalicular membrane function by diclofenac. Drug Metabolism and Disposition, v. 29, n. 12, p. 1535-1538, 2001.

16. KIRCHGESSNER, M. S. Meloxicam. Journal of Exotic Pet Medicine, v. 15, n. 4, p. 281-283, 2006.

17.SOUZA JÚNIOR, O. G.; LOURENÇO, A. S.; TABOSA, T. P. Análise histológica dos efeitos gástricos de aceclofenaco e meloxicam, estudo comparativo em ratos. Revista Paraense de Medicina, v. 15, n. 4, p. 28-32, 2001.

18. BAUCK, L.; BIHUN, C. Basic anatomy, physiology, husbandry and clinical techniques. In: HILLYER, E. V.; QUESENBERRY, 
K. E. Ferrets, rabbits and rodents. Philadelphia: Saunders, 1997. p. 291-306.

19.SÁNCHES, S.; ALARCON DE LA LASTRA, C.; ORTIZ, P.; MOTILVA, V.; MARTIN, M. J. Gastrointestinal tolerability of metamizol, acetaminophen, and diclofenac in subchronic treatment in rats. Digestive Diseases and Sciences, v. $47, \mathrm{n}$. 12, p. 2791-2798, 2002.

20.TILLEY, L. P.; SMITH, F. W. K. Consulta veterinária em 5 minutos. 2. ed. São Paulo: Manole, 2003. 1423 p.

21.GRAF, J.; RUMOR, C.; FONSECA, V. R. C. D. Hepatite causada pelo uso de diclofenaco sódico. Gastroenterologia Endoscopia Digestiva, v. 21, n. 2, p. 82-84, 2002.

22.SILVA, A. A. Avaliação clínica de Rattus novergicus após terapia com antiinflamatória com inibidor seletivo ou não para COX-2 por extrapolação alométrica, 2004. 93 f. Tese (Doutorado) - Universidade Federal de Santa Maria, Santa Maria, 2004.
23. CHAHADE, W. H.; GIORGI, R. D. N.; SZAJUBOK, J. C. M. Antiinflamatórios não hormonais. Einstein, v. 6, p. S166-S174, 2008. Suplemento 1 .

24.MEYER, D. J.; COLES, E. H.; RICH, L. R. Medicina de laboratório veterinária: interpretação e diagnóstico. São Paulo: Roca, 1995. 308 p.

25. KUMMER, C. L.; COELHO, T. C. R. B. Antiinflamatórios não esteróides inibidores da ciclooxigenase-2 (COX-2): aspectos atuais. Revista Brasileira de Anestesiologia, v. 52, n. 4, p. 498512, 2002.

26. KANEKO, J. J.; HARVEY, J. W.; BRUSS, M. L. Clinical biochemistry of domestic animals. 5. ed. San Diego: Academic Press, 1997. 932 p.

27.CARVAlHO, W. A.; CARVALHO, R. D. S.; SANTOS, F. R. Analgésicos inibidores específicos da ciclooxigenase-2: avanços terapêuticos. Revista Brasileira de Anestesiologia, v. 54, n. 3, p. 448-464, 2004. 